\title{
Citizen science in radiation research
}

\author{
Cecilia Gustavsson ${ }^{1, *}$, Erik Andersson-Sundén ${ }^{1,}$, Abigail Barker ${ }^{2}$, , Anders Hjalmarsson ${ }^{1,}$, Mattias Lantz ${ }^{1,}$, Karl Lundén $^{3,}$, \\ and Stephan Pomp ${ }^{1,}$ \\ ${ }^{1}$ Department of Physics and Astronomy, Uppsala University, Sweden \\ ${ }^{2}$ Department of Earth Sciences, Uppsala University, Sweden \\ ${ }^{3}$ Department of Forest Mycology and Plant Pathology, Swedish Agricultural University
}

\begin{abstract}
A growing trend in science is that research institutions reach out to members of the public for participating in research. The reasons for outreach are many, spanning from the desire to collect and/or analyse large sets of data efficiently, to the idea of including the general public on a very fundamental level in sciencemaking and ultimately decision-making. The presented project is curriculum-based and carried out in 240 lower secondary school classes (pupils of age 13-16). The task, as designed by the participating universities, is to collect mushrooms, soil and animal droppings from different parts of Sweden, do preliminary sample preparation and analyses and send the samples to the university institutions for radioactivity measurement. Behind the project is a desire to compare today's levels of ${ }^{137} \mathrm{Cs}$ with those deposited right after the Chernobyl accident in 1986, but also to study the exchange of caesium between organisms as well as the impacts of biological and geological processes on uptake and retention. The scientific outcome is a geodatabase with the ${ }^{137} \mathrm{Cs}$ activity $\left(\mathrm{Bq} / \mathrm{m}^{2}\right)$ present in the Swedish environment, where radioactivity data can be linked to the species (fungi, competing species, animals foraging), forest type, land type, land use and other environmental factors. The science question is of interest to the general public as foraging for mushrooms, as well as spending recreational time in forests is widely popular in Sweden. In this article, we will discuss the current status of the project and the observations we have made about how well the public can participate in scientific research. Focus will be on organization of the project, such as logistics, preparation of supportive material, feedback and communication between researchers and schools. We will present observations about the impact the project has had on the participants, based on quantitative and qualitative evaluations.
\end{abstract}

\section{Introduction}

Citizen science is a term used to describe research or scientific investigations which is either driven by members of the public, or where members of the public participate together with a research institution. Many citizen science projects are based on voluntary participation and do by default attract people already interested in science. A large number of such projects are coordinated by nature and wildlife organizations, e.g. [1] and [2] and aim at studying biodiversity such as counting of birds or butterflies. Furthermore, many universities and scientific research institutes offer citizen science projects within many different disciplines such as astronomy, biology, social sciences and humanities.

There are many motivations for setting up a citizen science project. One can simply wish a large number of people helping in collecting or analysing data, in order to obtain a large research material. However, it is very common that citizen science project also target other values, such as spreading knowledge of scientific processes, creating interest in nature or the research question itself, or enhancing the participants' sense of being part of a communal effort. Many studies, see eg. [3] and references therein, show that

*e-mail: cecilia.gustavsson@physics.uu.se participants in citizen science projects become more interested in and engaged in the research they are contributing to, and that they also feel connected to other people and to democratic and civil rights.

The obvious drawback of only attracting those already interested is that there are a lot of people that will not be reached. If one outspoken goal is to educate or spread knowledge about your research, a less exclusive approach then the purely voluntary is preferable. One of the types of citizen science projects that can be very rewarding, if feasible, is identified by Bonney et al. [4] as curriculumbased projects. This means involving school pupils at some level which gives the opportunity to reach a very wide and diverse group of young people. For some participants, such an experience will be their only contact with science, which makes the encounter even more important than for those who will spend their whole career doing science.

\section{Organisation of the project}

The project at hand is a curriculum-based citizen science project targeting pupils in the senior level of compulsory school in Sweden. The mandatory Swedish school system is nine years, and this project was directed to the years 7-9 
corresponding to pupils aged 13-16. The project was carried out during the autumn of 2018 and the task for the schools was to collect mushrooms, soil samples and animal droppings from their local environment and do sample preparation such as weighing, drying and dispersing the mushroom samples. They also had to identify the mushroom species as well as the animal droppings; in case they found it. Finally, all samples were sent to the Uppsala University for radioactivity measurements, primarily of the isotope ${ }^{137} \mathrm{Cs}$. The background to the investigation is the the Chernobyl accident in 1986 which deposited radioactive fall-out over large parts of Sweden.

Our project was organized in such a way that teachers signed up their class(es) using a web form, and since we had 250 entries to the project and the first 250 schools to register were accepted. We reached a good coverage over Sweden with the first come first served method, so no screening of the original entries was done.

\subsection{Preparation of supportive material}

In our curriculum-based citizen science projects, the teachers formed the link between the pupils and the university researchers. All communication was through the teachers and the supportive material was directed to them for further education of their pupils. Earlier research, [4], has shown that in order to be effective, it is crucial for a curriculum project to give adequate supportive material as well as suitable training to the teachers.

For all communication, an open website [5] was set up at the university server. Through this site all necessary material could be obtained and downloaded. The website contained basic background, had contact information, a FAQ-page and eventually a result page. Through it, the teachers could also book Skype-meetings with part-taking researchers.

At the website we provided a thorough teacher's guide with background facts about ionizing radiation, decay, fungi (mushroom) and our scientific idea. The guide also explained the experiment and tasks for the schools in great detail, as well as provided protocols for their work. Later, we asked the teachers in a questionnaire how they perceived the teachers' guide. The result can be seen in table 1. We had some worries that the guide would be too long and too difficult, but according to the answering teachers that was not the case. In total, the teachers' guide constituted of roughly 50 pages plus three different protocols for mushrooms, soil and droppings. In addition to the printable teachers' guide; a short film about radiation was produced, intended to be viewed by the classes as a teaser and start-up for the project.

The website and entry form was opened in May 2018. Once the 250 available places were filled, the participating schools were equipped with a research box containing all the equipment needed for their field work. The box contained plastic jars for storing samples, a metal rod for extracting soil samples, plastic gloves, a kit for DNA extraction and parts for assembling a simple radiation detector. Also pre-printed labels and pre-paid envelopes for posting the samples were included. The idea was that all that the

\begin{tabular}{l|ccc}
\hline with respect to & bad/difficult & adequate & good/easy \\
\hline clarity & 2 & 19 & 16 \\
complexity & 2 & 23 & 10 \\
length & 4 & 23 & 10 \\
\hline
\end{tabular}

Table 1: How did you as teacher perceive the teacher's guide?

schools needed for participating should be included in the research box with no extra costs attached.

\subsection{Feedback and communication}

As mentioned in section 2.1, the website was our communication channel. Teachers could book Skype meetings, an opportunity that was only exploited by a couple of schools. The schools were obliged to send in their samples by the end of October 2018. The summer of 2018 was unusually hot and dry in Sweden which unfortunately affected the amount of mushroom in early autumn. This could be one of the factors behind to fact that out of 250 sent out research boxes, we received back samples from 135 . It should however be noted that some schools with several participating classes merged their samples together. Nevertheless, we received in total 248 samples of dried mushroom (each class could send up to three samples), the same number of soil samples and around 50 samples of animal droppings.

The first step in our investigation was to measure the ${ }^{137} \mathrm{Cs}$ content of the dried mushrooms using a HPGe detector. This work was ongoing during November and December of 2018 and during this time we communicated with the schools by uploading short films on sample handling, measuring and storing. Guides to successful citizen science projects, e.g. [6] stress that feedback is a key point in maintaining the interest from the participants and acknowledging their work and effort. We thought it important to keep in contact with our schools during the long measurement time and produced four short films with the titles "The samples arrive in Uppsala", "How the detector works", "Measuring mushrooms" and "What the measurement showed". Participating teachers got notifications about these feedback efforts. All films can be accessed through the website [5]

\subsection{The mushroom sandwich index}

Once preliminary results were at hand, a final film with the name "We have a result" was uploaded to the project website. In this film we presented the preliminary results of ${ }^{137} \mathrm{Cs}[\mathrm{Bq} / \mathrm{kg}]$ in mushroom in the form of graphs and a Google map (can be reached from [5]) where each school class could find information about their own samples.

The unit $\mathrm{Bq} / \mathrm{kg}$ is difficult to grasp, and when communicating to the public, it is good to be able to compare the activity to something familiar. First of all, one usually talks about doses in the unit Sievert [Sv] which takes into account the type of ionizing radiation and the effect on human tissue. We have used a conversion factor from 
the International Commission on Radiological Protection (ICRP 119), [7], which for the digestion of ${ }^{137} \mathrm{Cs}$ is given as $1.3 \cdot 10^{-8} \mathrm{~Sv} / \mathrm{Bq}$. Next, one would like to compare the obtained doses to the dose given by something familiar. One common such quantity is the banana equivalent dose (BED), i.e. to compare a sample's dose to the activity of eating one average banana. The BED is quoted as $0.1 \mu \mathrm{Sv}$ [8].

For this work, however, we did not find the BED suitable, because it comes from the decay of ${ }^{40} \mathrm{~K}$ and has nothing to do with the decay we primarily wanted to investigate; the one from ${ }^{137} \mathrm{Cs}$. Instead, we constructed a "mushroom sandwich index" which illustrates how many mushroom sandwiches a person has to eat of a particular batch of mushrooms in order to get the dose $1 \mathrm{mSv}$ per year; a dose that the Swedish Radiation Safety Authority uses to assess risks. We have assumed that a mushroom sandwich contains $200 \mathrm{~g}$ of mushroom.

Such a presentation of the results was more intuitive and understandable for the school pupils than simply quoting the $\mathrm{Bq} / \mathrm{kg}$ of their samples. The mushroom sandwich index of every sent in sample was given as feedback on the Google map, together with the activity in $\mathrm{Bq} / \mathrm{kg}$. The indexes ranges from 0.5 mushroom sandwich per day (corresponding to $100 \mathrm{~g}$ of mushroom per day for the strongest sample) to more than 1000 sandwiches per day. Given the mushroom sandwich indexes we obtained, it is understood that one has to consume very large quantities of wild mushroom to reach an extra dose of $1 \mathrm{mSv}$ per year.

\section{Measuring the impact on participants}

When we designed this citizen science experiment, one of the main objectives was to reach out to a young audience with our research and plant interest and knowledge about scientific processes. In order to evaluate how they perceived the project and if it gave any impact, we constructed two questionnaires; one for the participating teachers and one for the pupils. The teachers' questionnaire was answered by 42 individuals, and the pupils' by 600 . In the following sections we will discuss some of the results from these questionnaires.

The questionnaires had multiple-choice questions, which are easy to evaluate quantitatively. Such questions are the bases for figures 1, 3-4 and tables 1-4

Furthermore, we asked questions that required freetext answers. The figures 2 and 5-7 are based on such questions. The quantitative analysis behind the representations was made in such a way that the free-text answers were assigned to different categories based on the occurrence of certain key words (or synonyms). Some answers contained several of the key words and were then counted in more than one category. In a few cases in the pupils' questionnaire, an answer was interpreted as unserious or unreliable and was then not counted. These answers could e.g. be random letters, swearwords or the like. This filtering and interpretation of written text is difficult and infers an uncertainty in the quantitative handling of the results. However, since most free-text answers are short (usually only one short sentence, or even just one word) it was in most cases not difficult to assign answers to categories. Many pupils in particular use the same words, such as (the Swedish translation of) fun, boring, instructive, etc.

\section{Results - teachers}

The first questionnaire was sent to the teachers who had signed up for our project, and as mentioned in section 3 it was answered by 42 individuals. In total there were 28 questions, including some follow-ups to other questions. For this article we have only analysed a few of these questions, and work on the full questionnaire including interesting correlations is on-going.

The first question we would like to discuss was phrased "Is it your opinion that the project has influenced the pupils' interest in science and technology?" which was answered by 35 teachers. The result can be seen in figure 1 .

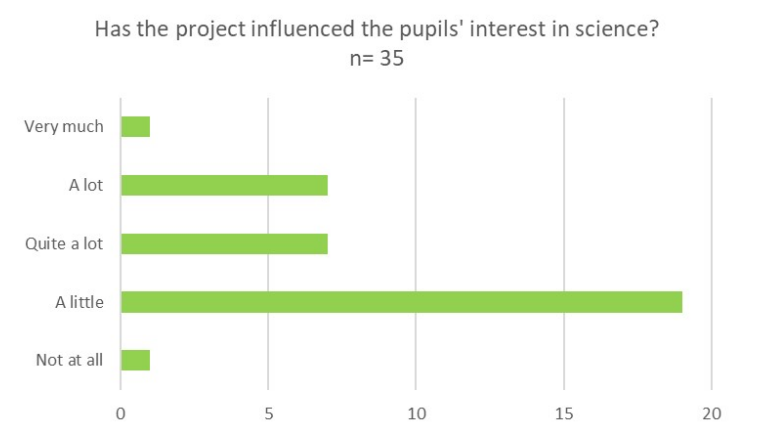

Figure 1. Question put to participating teachers about the projects' influence on pupil interest in science.

Interpreting the answer alternatives, only one says "no", while four answers are "yes", in various degrees. The translation from the Swedish original is difficult, there is a more stringent linguistic differences between the options translated to "a lot" and "quite a lot" in the original Swedish. Nevertheless, the result from this question shows that, according to these teachers, there was an impact on the pupil interest in science and technology. Giving the fact that most school classes spend only a few hours in total on the project, the the most common answer "a little", seems reasonable.

The next question we would like to present from the teachers' questionnaire is "Was it something the pupils particularly appreciated?", answered by 27 teachers. The results to this question is visualised in figure 2 .

Figure 2 shows that the two most appreciated things with the project was that it was "something real" and that it gave the opportunity to do "something different" in particular "being outdoors". To do something "real"; in this case take part in a scientific study, acts as a motivator, and it is well known that motivation is one of the strongest factors for learning. In this case, the pupils studied an area close to their homes and gained insight in the environmental situation with respect to radioactivity, and it is not hard to imagine that such a task it motivating in itself. Spending time in the forest and foraging for mushroom are popular 


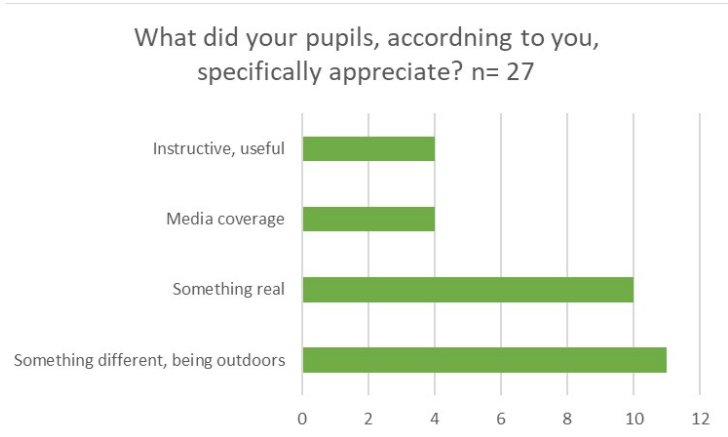

Figure 2. Question put to participating teachers about what the pupils particluarly appreciated.

recreation activities in Sweden, and it is clear that it is popular for pupils to do something different that spending all days in the classroom.

One can note the choice "media coverage" as an other factor that was appreciated. Our project gained interest from media, and some schools were visited by local press. The fourth choice; "instructive, useful" was also mentioned by a number of teachers, and we will return to that when we look at the pupils' responses.

\section{Results - pupils}

The pupils' questionnaire had 600 answers and the tables 2 and 3 show the gender and grade of the respondents respectively. In total, there were 16 questions in the questionnaire and only part of the material is presented here.

\begin{tabular}{ccc}
\hline \multicolumn{3}{c}{ Gender } \\
\hline female & male & other \\
295 & 276 & 29 \\
\hline
\end{tabular}

Table 2: Gender of the pupil respondents

\begin{tabular}{ccccc}
\hline \multicolumn{5}{c}{ Grade } \\
\hline 6 & 7 & 8 & 9 & other \\
30 & 223 & 202 & 119 & 22
\end{tabular}

Table 3: Grade of the pupil respondents

Figure 3 shows how the pupils replied to the question "Have you talked to someone outside your school class about the project?".

It can be seen from the figure 3 that a majority of the pupils state that they have talked to persons outside their class about the project. The most popular people to talk to are family and friends. The fact that a majority of our participating pupils have been talking about the project outside school shows that they have been thinking about and wanting to share thoughts about the project. It should be noted that for this question, several options could be chosen by the respondents.

One can try to assess how and if learning outcomes were obtained in a citizen science project, and previous

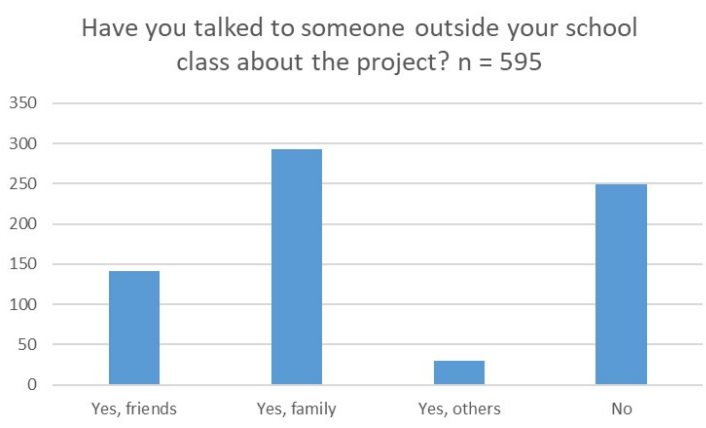

Figure 3. Question put to the pupils about if they have talked to others about the project.

studies such as [4] and [9] suggest that participation in a citizen science project does lead to enhanced knowledge about the subject of the study. In our questionnaire to the pupils, we asked the questions "Did you before the project know how to measure radioactivity" and " Do you after the project know how to measure radioactivity". The first question was answered by 593 pupils and the second by 591, and the results can be seen in figure 4 .

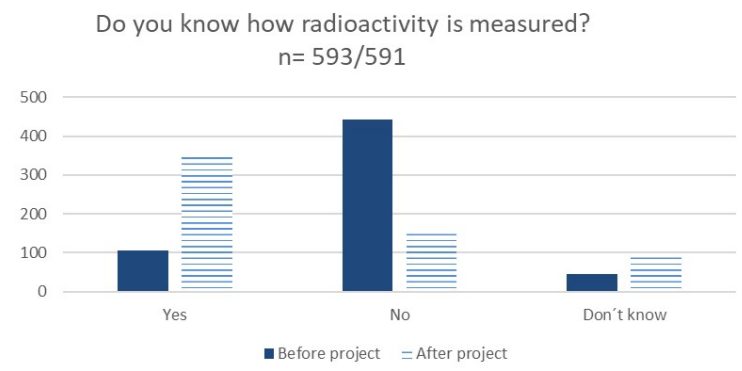

Figure 4. This figure is based on two questions asking the pupils if they knew before and if the know after the project how radioactivity is measured.

The results of these two questions show very clearly that the number of pupils answering "yes" to the questions is significantly larger when its about their knowledge after the project than before, and the number answering "no" is significantly lower. It is interesting that also the "don't know" reply is more common after the project. We believe that the reason that many pupils having learnt how radioactivity is measured is due to the fact that we provided a lot of material on the topic and shorts films from our measurements, but also the fact that one of their tasks was to assemble and measure with a simple radiation detector.

We also asked the pupils what it was like to participate, see figure 5. These replies are based on categorizing key words, as explained in section 3 and some answers are counted in several categories. 410 individual responded to the question.

It is nice to see that the most common answers were good or fun (forming one category) followed by exciting, interesting or instructive (forming another category). The word "boring" was mentioned 69 times and fun or good 203, making that option almost 3 times more com- 


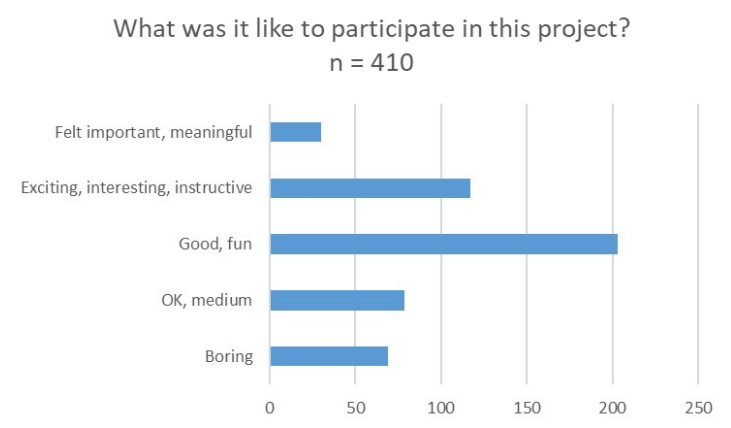

Figure 5. Question to the pupils about what it was like to participate in the project. Some pupils give multiple answers that fall in several categories.

mon. There was an overlap between fun/good and exciting/interesting/instructive, but also an overlap between boring and e.g. instructive. These correlations have not been explored in detail and the analysis is on-going.

From the 203 answers saying fun or good, 86 had stated why, see figure 6. Pupils wrote e.g. "it was fun because we could be outdoors", or "it was fun because I learned a lot".

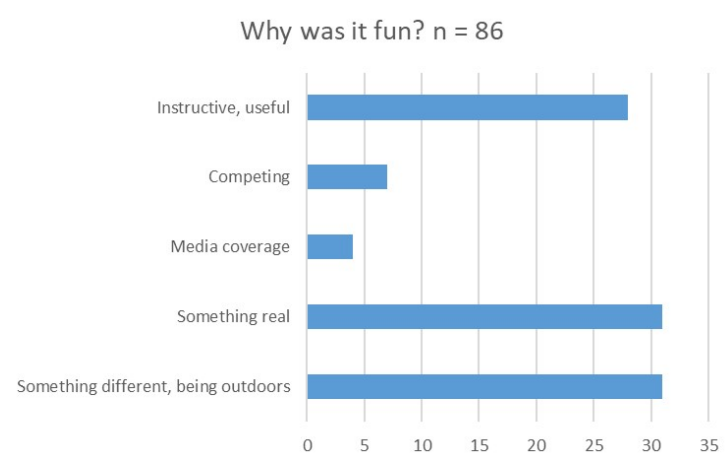

Figure 6. Questions to the pupils following up on those who answered that it was fun to participate in the project.

Some pupils found the competition extra motivating and fun; there was an option for the school classes to compete with representation of their work in the form of e.g. a poster or a film which was obviously popular. The winning contribution was rewarded with a sum of money.

Comparing figure 6 to figure 2 , it stands out that teachers and pupils agree that important factors they appreciate are being outdoors and doing something real. But it also seems that the pupils value the instructiveness and usefulness higher than the teachers believe. It is an important finding and an interesting thing to follow up on.

There is a lack of evidence in the literature, that citizen science actually impact the participants attitude towards science [9]. We tried to investigate this topic by asking the pupils if they had imagined themselves doing science before the project and now after. Results can be seen i table 4 .

As can be seen, also in our project there is little evidence that participating changed the pupils' view on their

\begin{tabular}{l|ccc}
\hline & yes & no & maybe \\
\hline females before & 35 & 188 & 66 \\
females after & 27 & 174 & 91 \\
\hline males before & 49 & 150 & 71 \\
males after & 25 & 152 & 99 \\
\hline
\end{tabular}

Table 4: Question to the pupils: Have you imagined yourself doing science?

own possibilities of doing science. We did correlate this question with gender, but for both males and females the only significant difference between before and after is that group answering "maybe" has become larger. That is an interesting find, and could be interpreted that the pupils after their experience have become more uncertain or changed their view on what science is, a finding that is not necessarily negative.

Finally we asked the pupils if they would like to become researcher themselves, and what they would like to do research on. 70 pupils replied and stated their research interest, see figure 7 .

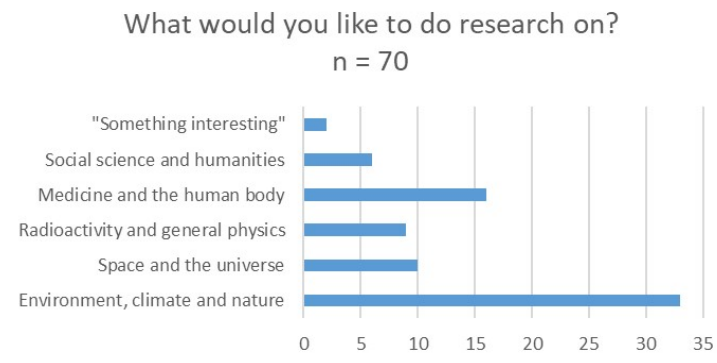

Figure 7. Pupils' replies to the question what they would like to do research on.

Also this question was answered by text answers, and they were treated by sorting into categories as has been described before. The two mostly occurring topics were environment/climate research and medicine. The third most common reply was space and the universe.

\section{Discussion and outlook}

This has been a very rewarding project and a lot of interesting science is coming out. The results on radioactivity in mushroom is presented elsewhere, and analysis is going on regarding radioactivity in soil and transport mechanisms between soil, mushrooms and other organisms. Also evaluating the citizen science part of the project requires more in-depth studies of the material and more sophisticated qualitative analysis methods. This work is on-going.

When handling the participant questionnaires, one must take the answering frequency and possible biases into account. All participants had the opportunity to fill in the reply forms, but 42 teachers out of 135 classes constitute only $31 \%$. One can note however, that many teachers had several classes participating and some classes had several teachers so the answering frequency is hard to calculate exactly. For the pupils, it is impossible to know 
how many participated. There are very large differences in class sizes in Swedish schools, ranging up to a maximum of 30 pupils per class; so a maximum number of participating pupils would be around 4700. However, 25 pupils per class is probably a more realistic average, giving a number of 3400 . In that case, the answering frequency is around $18 \%$. One must also assume, that some teachers who answer the questionnaire also encourage their pupils to do so, leading to a certain bias based on mutual experiences. It can also be assumed that those teachers who enjoyed taking part are more likely to answer the questionnaire and encourage their pupils to do the same.

\section{Acknowledgement}

The authors would like to thank the unit for Communication and school collaboration at the Faculty of Science and Technology, Uppsala University, for selecting this project as a citizen science project. The Swedish Radiation Safety Authority has supported the project financially

\section{References}

[1] https://www.birds.cornell.edu/home/about/

[2] https://butterfly-conservation.org/in-yourarea/european-butterflies-group

[3] Dayer, A. et al., People and Nature 1, 138-151 (2019)

[4] Bonney, R. et al., Public Understanding of Science 25(1), 2-16 (2015)

[5] http://teknat.uu.se/samverkan/skola/massexperiment/stralandejord/

[6] Tweddle, J.C et al., Natural History Museum and NERC Centre for Ecology and Hydrology for UK-EOF (2012)

[7] Eckerman, K., et al., Annals of the ICRP 42(4), 1-130 2013)

[8] Usually attributed to Gary Mansfield of the Lawrence Livermore National Laboratory, 1995

[9] Brossard D., et al., International Journal of Science Education 27(9), 1099-1121 (year) 